\title{
Valor percibido y lealtad del cliente: estrategia cobranding de tarjetas de crédito en Bogotá (Colombia)*
}

\author{
Sara Catalina Forero-Molina** \\ Samir Ricardo Neme-Chaves***
}

Fecha de recibido: 10 de julio de 2020 Fecha de aprobado: 7 de octubre de 2020

Para citar este artículo: Forero-Molina, S. C., \& Neme-Chaves, S. R. (2021). Valor percibido y lealtad del cliente: Estrategia cobranding de tarjetas de crédito en Bogotá (Colombia). Revista Universidad E Empresa, 23(40), 1-18. https://doi.org/10.12804/ revistas.urosario.edu.co/empresa/a.9335

\section{Resumen}

El cobranding es una oportunidad estratégica de cooperación entre marcas, al punto que las involucradas en la alianza buscan beneficios particulares sin perder su identidad. Existen múltiples tipos de cobranding, uno de los cuales es el cooperativo o de alcance y conocimiento, del que hace parte la estrategia de tarjetas de crédito de marca compartida. Se trata de un tipo de alianza creciente en los últimos años en Colombia con diversas pretensiones, como la incursión en nuevos nichos de mercado, nuevos beneficios

* La investigación presentada en este artículo fue financiada por la Universidad Santo Tomás (Colombia), a través del Acta 18130040, otorgada a los dos autores, y llevó el título "Análisis de la percepción de valor y la lealtad de los clientes de las tarjetas de crédito de marca compartida para la categoría institución bancaria-tienda minorista de ropa, accesorios y calzado en Bogotá".

** Magíster en Administración de la Universidad Nacional de Colombia. Docente investigadora de la Facultad de Mercadeo, Universidad Santo Tomás (Colombia). Correo electrónico: saraforero@usantotomas.edu.co. ORCID: https:// orcid.org/0000-0002-3020-0690

*** Magíster en Psicología del Consumidor de la Fundación Universitaria Konrad Lorenz (Colombia). Docente investigador de la Facultad de Mercadeo, Universidad Santo Tomás (Colombia). Correo electrónico: samirneme@usantotomas.edu.co. ORCID: https://orcid.org/0000-0003-2327-4947 
para los clientes, mayor fidelización de los clientes, entre otros. En conformidad, la investigación tuvo como objetivo identificar la relación entre la percepción de valor hacia las tarjetas de crédito de marca compartida y la lealtad hacia marcas de ropa, accesorios y calzado en Bogotá (Colombia). Se llevó a cabo un estudio cuantitativo de corte correlacional con diseño transversal, contando con un muestreo no probabilístico-por conveniencia con un total de 250 encuestas válidas. Los resultados dejan entrever una relación entre los factores de valor percibido hacia las tarjetas de crédito de marca compartida y los factores de lealtad hacia las marcas estudiadas, y se pudieron validar todas las hipótesis planteadas.

Palabras clave: percepción de valor; lealtad; tarjetas de crédito de marca compartida.

\title{
Perceived Value and Customer Loyalty: Credit Card Co-branding Strategy in Bogotá - Colombia
}

\begin{abstract}
Co-branding is a strategic opportunity for cooperation between brands. Those involved in the alliance seek particular benefits without losing their identity. There are multiple types of co-branding; one is cooperative or outreach and knowledge, which is part of the co-branded credit card strategy. It is a growing type of alliance in recent years in Colombia with various claims for brands such as the incursion into new market niches, new benefits for customers, and increased customer loyalty, among others. In accordance, the present investigation aims to identify the relationship between the perception of value towards credit cards of the shared brand and the loyalty towards the clothing, accessories, and footwear brands in Bogotá-Colombia. A cross-sectional correlational quantitative study was carried out, with a non-probability sampling — for convenience with a total of 250 valid surveys. The results hint that there is a relationship between the perceived value factors towards shared brand credit cards and the loyalty factors towards the studied brands, thus being able to validate all the hypotheses proposed.
\end{abstract}

Keywords: Perceived value; loyalty; co-branded credit cards.

\section{Valor percebido e lealdade do cliente: estratégia de co-branding de cartões de crédito em Bogotá-Colômbia}

\section{Resumo}

A prática de co-branding é uma oportunidade estratégica de cooperação entre marcas, no sentido de que, as partes envolvidas na aliança buscam benefícios particulares sem perder a própria identidade. Existem vários tipos de co-branding, um deles é o cooperativo ou de alcance e conhecimento, do qual fazem parte empresas de cartões de crédito de marcas compartilhadas. Trata-se de um tipo de aliança crescente nos últimos anos na Colômbia, cujas marcas possuem diversas pretensões, como a entrada em novos nichos de mercado, novos benefícios para os clientes, maior fidelização dos clientes, entre outros. Nesse sentido, a presente pesquisa tem como objetivo identificar a relação entre a percepção de valor dos cartões de crédito de marcas compartilhadas, assim como a fidelidade para com marcas de roupa, acessórios e calçados em Bogotá - Colômbia. Procedeu-se um estudo quantitativo correlacional e transversal, com uma amostra não probabilística - por conveniência com um total de 250 pesquisas de opinião válidas. Os resultados demonstram a existência de relação entre os fatores de valor percebido em relação aos cartões de crédito de marcas compartilhadas e os fatores de fidelidade em relação às marcas estudadas, validando dessa forma, todas as hipóteses levantadas.

Palavras-chave: percepção de valor, lealdade, cartões de crédito de marca compartilhada. 


\section{Introducción}

El cobranding se ha constituido en una oportunidad estratégica de cooperación entre marcas, a partir de la cual aquellas involucradas en la alianza están en la búsqueda de beneficios particulares, sin perder la identidad de cada una y generando valor añadido. En términos generales, el cobranding tiene como meta el desarrollo efectivo de lealtad en los clientes para el producto resultado de la alianza o para las marcas de forma individual (Kim et al., 2007) y la generación de favorables índices de valor percibido en los clientes (Helmig et al., 2008). Así, se ha transformado en cierto sentido la concepción de la competencia entre marcas y se ha adaptado a la de cooperación (Grębosz, 2012; Grębosz-Krawczyk \& Pointet, 2017), al observar que, como afirman Swaminathan et al. (2015), permite respuestas más positivas de los consumidores.

En ese orden de ideas, el cobranding se fundamenta en el sentido de la alianza, lo que implica que se comparten objetivos, desde el entendimiento de que las marcas buscan alianzas, ya que "ninguna compañía puede movilizar todos los recursos y activos necesarios para responder a las rápidas oportunidades de negocios una y otra vez" (Rúmbolo, 2015, p. 15), por lo que es conveniente buscar formas de colaboración efectivas. Esto es consistente con la apreciación de Kapferer (2008) respecto a que "el co-branding es el resultado de un deseo de permanecer dentro de las competencias clave de la empresa, hasta el punto de buscar en otras partes las competencias que faltan" (p. 166).

Lo anterior constituye un reto significativo para las marcas que deciden implementar el cobranding, en la medida en que no se trata de generar confusión en el mercado en cuanto a su identidad y personalidad, sino precisamente en reforzarlas. Esto basado en el hecho de que la evaluación del consumidor hacia las marcas asociadas se puede ver alterada (para beneficio o perjuicio) por la exposición a la alianza, en lo cual influyen diversos factores como las actitudes preexistentes hacia las marcas, el ajuste percibido de los productos y el ajuste percibido de las marcas (Simonin \& Ruth, 1998; Bravo \& Pina, 2009). Ello implica el riesgo de pérdida de credibilidad o no elevación de la calidad percibida de una de las marcas de la alianza (Park et al., 1996; Rúmbolo, 2015; Tur Viñes, 2012). 
Es preciso evidenciar que la intensa competencia en los mercados saturados actuales, especialmente en lo referente a los bienes de consumo de rápido movimiento, ha llevado a que el uso de productos cobranded sea cada vez más importante (Helmig et al., 2008). Si bien existen diferentes tipos de cobranding, en términos generales, Hoyos (2016), apoyado en Lamb et al. (2011) y en Blackett y Boad (2011), menciona cuatro: a) de ingredientes, cuando un producto (marca senior) incluye un ingrediente o se combina con otra marca (marca junior); b) cooperativo o de alcance y conocimiento, concentrado en intangibles, donde las marcas que se unen buscan desarrollar sinergia utilizando lo principal de su saber hacer con el principal objetivo de obtener clientes de forma rápida y segura, aumentando así los ingresos; c) basado en el respaldo de valores, orientado a una causa, y d) de marcas complementarias, no existe la marca senior y la junior, las dos tienen la misma importancia.

Respecto al sector retail y cobranding, es posible encontrar los estudios de Dahlstrom y Conway Dato-On (2004), en los cuales se evaluaron los factores y los antecedentes importantes y potenciales ante la estrategia de cobranding para el sector minorista; el de Ching Wang et al. (2015), en el que se interpreta la reacción de dos culturas diferentes ante la estrategia cobranding retailer para marcas de lujo; el de Wright et al. (2007), que investiga el uso del cobrading en franquicias, enfocando en un estudio de caso hacia McDonald's/McCafe, y formulan un modelo teórico; el de Shen et al. (2014), así como el de Oeppen y Jamal (2014), en el cual se examina empíricamente el cobranding en el sector de la moda rápida y sus efectos, por mencionar algunos.

Por otra parte, se aprecia el auge que han venido teniendo en el mundo las tarjetas de crédito de marca compartida o productos de alianza cobranding entre marcas de retail y bancarias. ElBoghdady (2003) manifiesta que los acuerdos de cobranding para las tarjetas florecieron entre 1992 y 1999, aun cuando se estancaron por la baja rentabilidad que representaron para algunos bancos y resurgieron posteriormente bajo la premisa de generar distinción en un mercado altamente competitivo.

Así, es interesante detallar el crecimiento de estas estrategias cobranding en los últimos años en Latinoamérica, y que para el caso colombiano, según la Superintendencia Financiera de Colombia (2019), representa un total de 15420376 tarjetas de crédito vigentes para abril, y de acuerdo con la misma fuente (citada en El Universal, 2016), para diciembre 
de 2014, de los 12684370 unidades de tarjetas de crédito en circulación, 2290819 correspondían a alianzas cobranding entre el sector financiero y las grandes cadenas de retail del país. Se destaca como el banco pionero a Colpatria, en cuanto a la implementación de tarjetas de marca compartida hacia los años noventa, y que hoy ocupa el segundo puesto de este tipo de productos, seguido por el banco Davivienda. Otras entidades bancarias que cuentan con estas tarjetas en Colombia, son Banco de Occidente, Banco de Bogotá, Scotiabank Colpatria, AV Villas, Bancolombia, BBvA, Banco Caja Social y Helm Bank.

En la práctica, se argumentan diferentes motivos por los cuales son beneficiosas las tarjetas de alianza entre entidades bancarias y el sector retail. Para las entidades bancarias, esta estrategia posibilita incursionar en nuevos nichos de mercado, mejorar los beneficios para los clientes, generar a los clientes exclusividad en la adquisición de productos e incrementar en sí el uso de la tarjeta; para el sector retail, los beneficios giran en torno a que permiten lograr mayor fidelización entre los clientes, atraer nuevos, garantizar que seguirán visitando el comercio y comprando y generar recordación de marca.

A nivel académico se destaca el estudio de Wang y Hsu (2016), el cual deja entrever que existe una relación positiva y significativa entre la actitud de los consumidores hacia las tarjetas de crédito de marca compartida de aerolínea, los beneficios percibidos, las normas subjetivas y el control de la conducta percibida. Por su parte, el estudio de Wang y Farquhar (2018) demostró una fuerte asociación entre los beneficios percibidos asociados por parte de los consumidores y la intención de usar una tarjeta de crédito de marca compartida, teniendo como variable moderadora el involucramiento.

Teniendo en cuenta lo anterior, se planteó como objetivo para la investigación identificar la relación entre la percepción de valor hacia las tarjetas de crédito de marca compartida y la lealtad hacia marcas de ropa, accesorios y calzado en Bogotá. Así, se constituye en un aporte, pues no existe literatura académica específica para interpretar este tipo de estrategia cobranding en el país, y la evidencia empírica también es poca, ya que no se ha indagado por el impacto y los resultados de forma explícita, más allá de lo que se refiere a la cuota de mercado y, como lo refieren Wang y Hsu (2016), es limitada la evidencia de investigación empírica sobre la efectividad de la marca compartida en la práctica. 
A continuación se contextualiza lo referente a valor percibido y lealtad. El primero es "la evaluación global del consumidor con respecto a la utilidad del producto basada en la percepción de lo que se recibe a cambio de lo que se da" (Zeithaml, 1988, p. 14); el concepto está arraigado en la teoría de la equidad, donde un consumidor evalúa el costo de un producto en comparación con los beneficios recibidos (monetarios y no monetarios) (Bolton \& Lemon, 1999; Yang et al., 2011). Los consumidores deben percibir la transacción como justa y equitativa, lo que a su vez tendrá un impacto positivo en el comportamiento de compra. Así, Petrick (2002) propone una tipología del valor percibido que incluye cinco categorías: el valor emocional, el valor social, el valor utilitario, la calidad y el precio, siendo altamente valorada y utilizada por la comunidad científica (Dedeoglu et al., 2018; Kim et al., 2018; Nguyen, 2018).

Particularmente, el valor utilitario se define como "la utilidad percibida adquirida a partir de la capacidad de una alternativa para el desempeño funcional o físico. Una alternativa adquiere valor funcional a través de la posesión de atributos funcionales, utilitarios o físicos sobresalientes" (Sheth et al., 1991, p. 161). El valor social se define como "la utilidad percibida adquirida a partir de la asociación de una alternativa con uno o más grupos sociales específicos. Una alternativa adquiere valor social a través de la asociación con grupos demográficos, socioeconómicos y étnico-culturales estereotipados positiva o negativamente" (p. 161). El valor emocional se define como "la utilidad percibida adquirida de la capacidad de una alternativa para despertar sentimientos o estados afectivos. Una alternativa adquiere valor emocional cuando se asocia con sentimientos específicos o cuando precipita o perpetúa esos sentimientos" (p. 161). El valor utilitario "resulta de la búsqueda consciente de una consecuencia específica, las cosas pueden demostrar ser buenas, al mostrarse como un medio para algo que se admite que es bueno" (Babin et al., 1994, p. 646).

El valor hedónico resulta más de la diversión que de la finalización de la tarea (Holbrook \& Hirschman, 1982). Por tanto, "el valor de compra hedónico refleja el entretenimiento potencial y el valor emocional de las compras" (Babin et al., 1994, p. 645). El precio es la utilidad derivada del producto debido a la reducción de la cantidad de dinero que se paga por él (Sweeney \& Soutar, 2001). La utilidad es aquella que se deriva de la calidad percibida, y el rendimiento esperado del producto (Sweeney \& Soutar, 2001). Así, la calidad hace referencia a la percepción que el producto que se adquiere o se usa está bien 
constituido, es confiable y brinda las prestaciones esperadas (Petrick, 2002; Walsh et al., 2014). Por otra parte, Ramírez Angulo y Duque Oliva (2013) definen la lealtad como:

[...] una variable multidimensional que describe el comportamiento y la actitud de las personas, en relación a la preferencia de productos, marcas o establecimientos determinados por encima de otros de su misma clase. [Por tanto...] se refleja en el incremento de la probabilidad de repetir los comportamientos de compra y consumo de los mismos productos o servicios que han sido adquiridos, consumidos o usados con anterioridad. (p. 305)

En tal sentido, Oliver (1999, citado en Vera \& Trujillo, 2009) plantea que la lealtad es un fenómeno multifactorial y además se compone de lealtad cognitiva, afectiva, intencional y acción. Para Jacoby y Kiner (1973, citados en Ramírez Angulo \& Duque Oliva, 2013) la lealtad incluye: lealtad comportamental, afectiva y cognitiva. Como indican los autores, la primera se asocia con la frecuencia de compra y recompra; la segunda, con el vínculo emocional que tienen los consumidores hacia las marcas, y la tercera, con la atención y concentración que da una persona a sus procesos de consumo y elección de marcas. Esto quiere decir que la lealtad incluye aspectos psicológicos de evaluación y toma de decisiones, los cuales conllevan actitudes y emociones respecto a una marca o grupo de marcas de la misma categoría y, posteriormente, se convierten en comportamientos de compra efectivos y repetitivos (Jacoby \& Kyner, 1973; Berné, 1997; Delgado, 2004, citados en Colmenares \& Saavedra, 2007). De acuerdo con Rundle-Thiele (2005) y Söderlund (2006), citados en García y Fernández (2016), es posible que cada consumidor presente estados de lealtad en mayor o menor grado para cada una de las dimensiones, es decir, que por ejemplo se evidencie en un consumidor alta fidelidad comportamental pero baja actitudinal.

En general, se ha encontrado que existe una relación entre el valor percibido y la lealtad en diferentes productos, servicios o actores de la cadena de valor como ventas minoristas (Grace \& O'Cass, 2005; Mencarelli \& Lombart, 2017). El estudio de Jiang et al. (2018) deja entrever que la lealtad a la marca se ve influenciada por la experiencia favorable con las actividades de comunicación de marketing de la marca; entonces, si las actividades de comunicación se llevan a cabo durante la etapa previa al consumo, además generará respuestas afectivas positivas para mejorar la preferencia de marca que contribuya a aumentar el valor percibido. Así, el valor percibido es un mejor predictor de 
lealtad actitudinal y lealtad comportamental (número de visitas y ventas totales) que la satisfacción (Mencarelli \& Lombart, 2017).

Particularmente, el estudio de Shen et al. (2017) sobre alianzas cobranding entre diseñadores de lujo y marcas de moda rápida deja entrever que la alianza es beneficiosa para ambas marcas y es posible aumentar las lealtades de los clientes generando maximización de beneficios. De igual manera, evidenció que si la marca compartida tiene éxito, la lealtad a la marca de moda rápida debería ser alta; sin embargo, la revisión de la literatura no mostró estudios que hubiesen abordado la relación entre la percepción de valor y la lealtad en tarjetas de crédito de marca compartida, y es justo allí donde este estudio quiere aportar.

\section{Metodología}

La presente investigación es de carácter cuantitativo de corte correlacional con diseño transversal. El instrumento de recolección fue un cuestionario diseñado con una escala tipo Likert de 5 puntos, la cual tuvo un alfa de Cronbach de 0.8 y un Lambda 6 de Guttman de 0.83. Los datos se analizaron con el software $\mathrm{R}$ versión 3.6.1 (R Core Team, 2019). Se determinó un muestreo no probabilístico por conveniencia, se usó este muestreo por facilidad de acceso a la muestra. El trabajo de campo se llevó a cabo en el segundo semestre del 2018 y se obtuvieron un total de 250 encuestas recogidas virtualmente, a través de correo electrónico y redes sociales. Los criterios de inclusión fueron que el participante tuviera activa hace al menos 6 meses alguna de las tarjetas de crédito de marca compartida listadas, las cuales eran 9 tarjetas que circulan en el mercado bogotano para la categoría en estudio (institución bancaria-marca de ropa, accesorios y calzado). En tal medida, la tabla 1 indica las variables estudiadas y el enfoque de la escala. 
Tabla 1. Variables y escalas

\begin{tabular}{rlrr}
\hline Constructo & \multicolumn{1}{c}{ Subvariables } & \multicolumn{1}{c}{ Escala } & Nivel de medición \\
\hline Valor percibido & - Valor emocional & Elaboración propia con base en las defini- & Intervalo-escala Likert \\
& - Valor utilitario & ciones de Petrick (2002) & \\
& - Valor social & & \\
& - Precio & & \\
& - Calidad & & \\
\hline Lealtad & - Lealtad comportamental & Ramírez Angulo y Duque Oliva (2013) & Intervalo-escala Likert \\
& - Lealtad cognitiva & & \\
& - Lealtad afectiva & & \\
\hline
\end{tabular}

Fuente: elaboración propia.

Basado en lo anterior, la tabla 2 relaciona las hipótesis planteadas. Es preciso recordar que el valor percibido se enfoca en la tarjeta de crédito de marca compartida, y la lealtad, hacia la marca de ropa, calzado y accesorios.

Tabla 2. Hipótesis

\begin{tabular}{lll}
\hline Hipótesis & \multicolumn{2}{c}{ Relación positiva entre } \\
\hline $\mathrm{H} 1$ & Valor social & Lealtad comportamental \\
\hline $\mathrm{H} 2$ & Valor social & Lealtad cognitiva \\
\hline $\mathrm{H} 3$ & Valor social & Lealtad afectiva \\
\hline $\mathrm{H} 4$ & Valor emocional & Lealtad comportamental \\
\hline $\mathrm{H} 5$ & Valor emocional & Lealtad cognitiva \\
\hline $\mathrm{H} 7$ & Valor emocional & Lealtad afectiva \\
\hline $\mathrm{H} 8$ & Precio & Lealtad comportamental \\
\hline $\mathrm{H} 9$ & Precio & Lealtad cognitiva \\
\hline $\mathrm{H} 10$ & Precio & Lealtad afectiva \\
\hline $\mathrm{H} 11$ & Calidad & Lealtad comportamental \\
\hline $\mathrm{H} 12$ & Calidad & Lealtad cognitiva \\
\hline $\mathrm{H} 13$ & Calidad & Lealtad afectiva \\
\hline $\mathrm{H} 14$ & Valor utilitario & Lealtad comportamental \\
\hline $\mathrm{H} 15$ & Valor utilitario & Lealtad cognitiva \\
\hline & Valor utilitario & Lealtad afectiva \\
\hline
\end{tabular}

Fuente: elaboración propia. 


\section{Resultados}

En cuanto a los resultados, estos se dividen en cuatro partes: la primera tiene que ver con la caracterización de la muestra; la segunda, con las prácticas de consumo de los actuales clientes de las tarjetas de crédito de marca compartida estudiados para la categoría seleccionada; la tercera, con los niveles de lealtad y valor percibido, y la cuarta, con el análisis correlacional entre los constructos valor percibido y lealtad.

En primer lugar, la muestra se caracterizó por ser $53 \%$ mujeres y $47 \%$ hombres. El $24 \%$ tiene entre 20 y 26 años; el 27\%, entre 27 y 33 años; el 31\%, entre 34 y 40 años, y el 18\%, más de 40 años. La media fue 33.46 años; la desviación estándar, de 8.17, y el rango, de 20-55. Respecto a la ocupación, el 12\% son estudiantes; el 72\%, empleados; el $13 \%$, independientes, y el 3\%, amas de casa. En cuanto al estrato, el $2 \%$ son de estrato 2; el $25 \%$, de estrato 3; el $47 \%$, de estrato 4 ; el $18 \%$, de estrato 5 , y el $8 \%$, de estrato 6 . Con referencia al nivel de escolaridad, el $1 \%$ estudiaron hasta primaria; el 5\%, secundaria; el $4 \%$, carreras técnicas-tecnológicas; el 54\%, carreras profesionales, y 36\%, posgrados.

En segundo lugar, se realizaron cuatro preguntas sobre prácticas de consumo, lo cual indica que el 16\% de los encuestados adquirió la tarjeta de crédito de marca compartida hace "menos de dos meses"; el 16\%, "entre dos y seis meses"; el 34\%, "entre seis y doce meses", y el restante $34 \%$, hace "más de un año". En cuanto al interrogante sobre cada cuánto hace uso de la tarjeta en términos generales, el 29\% de los encuestados manifestó que "una vez al mes"; el 11\%, "entre 2 y 3 veces al mes"; el 11\%, "más de 3 veces al mes"; el 29\%, "una vez cada dos meses", y el 20\%, "una vez cada seis meses". En cuanto a la frecuencia de uso de la tarjeta para comprar en la marca de ropa propiamente, el $42 \%$ manifestó que "siempre"; el 14\%, que "casi siempre"; el 33\%, que "algunas veces"; el 10\%, que "casi nunca", y el $2 \%$, que "nunca".

También se interrogó sobre los motivos por los cuales decidió adquirir la tarjeta de crédito de marca compartida, lo cual arrojó que el 38\% la adquirió "porque me da crédito para comprar en esta marca"; el 24\%, "porque me gusta la entidad bancaria que la respalda"; el 28\%, "por los beneficios adicionales que brinda la tarjeta para esta marca", y el 14\%, "por los beneficios adicionales que brinda la tarjeta aparte de los de esta marca". 
En tercer lugar, en la tabla 3 se pueden observar los niveles de valor percibido y lealtad por factor y en consolidado. En valor percibido, el factor con la media más baja es el emocional, con 3.40, y el más alto es calidad percibida, con 4.09. El valor percibido en general tiene una media de 3.67. En cuanto a la lealtad, la media más baja corresponde a la cognitiva (3.55), y la más alta, a la afectiva (3.79). La lealtad en general tiene una media de 3.75 .

Tabla 3. Niveles de valor percibido y lealtad

\begin{tabular}{lcccccccc}
\cline { 2 - 8 } & \multicolumn{3}{c}{ Valor percibido } & \multicolumn{5}{c}{ Lealtad } \\
\cline { 2 - 8 } & $\begin{array}{c}\text { Valor } \\
\text { emocional }\end{array}$ & $\begin{array}{c}\text { Valor } \\
\text { utilitario }\end{array}$ & $\begin{array}{c}\text { Calidad } \\
\text { percibida }\end{array}$ & Precio & $\begin{array}{c}\text { Valor } \\
\text { social }\end{array}$ & $\begin{array}{c}\text { Lealtad } \\
\text { afectiva }\end{array}$ & $\begin{array}{c}\text { Lealtad } \\
\text { comportamental }\end{array}$ & $\begin{array}{c}\text { Lealtad } \\
\text { cognitiva }\end{array}$ \\
\hline $\begin{array}{l}\text { Media por } \\
\text { factor }\end{array}$ & 3.40 & 3.56 & 4.09 & 3.80 & 3.49 & 3.79 & 3.89 & 3.55 \\
\hline $\begin{array}{l}\text { Media por } \\
\text { constructo }\end{array}$ & & 3.67 & & & & 3.75 & \\
\hline $\begin{array}{l}\text { Desviación } \\
\text { estándar }\end{array}$ & 0.45 & 0.45 & 0.57 & 0.65 & 0.98 & 0.67 & 0.80 & 0.66 \\
\hline
\end{tabular}

Fuente: elaboración propia.

En cuarto lugar, se evaluó la relación entre las subvariables de los constructos valor percibido y lealtad de marca a partir del coeficiente de correlación de Pearson con un valor de significancia de 0.001. Como se aprecia en la tabla 4, todas las hipótesis se aceptan, lo que indica una relación positiva entre todas las dimensiones de valor percibido hacia las tarjetas de crédito de marca compartida y las de lealtad hacia las marcas de ropa.

Tabla 4. Correlaciones entre valor percibido y lealtad de marca

\begin{tabular}{lllll}
\hline Hipótesis & & Relación positiva entre & Correlación & Conclusión \\
\hline $\mathrm{H} 1$ & Valor social & Lealtad comportamental & 0.42 & Se acepta \\
\hline $\mathrm{H} 2$ & Valor social & Lealtad cognitiva & 0.33 & Se acepta \\
\hline $\mathrm{H} 3$ & Valor social & Lealtad afectiva & 0.27 & Se acepta \\
\hline $\mathrm{H} 4$ & Valor emocional & Lealtad comportamental & 0.19 & Se acepta \\
\hline $\mathrm{H} 5$ & Valor emocional & Lealtad cognitiva & 0.26 & Se acepta \\
\hline $\mathrm{H} 6$ & Valor emocional & Lealtad afectiva & 0.18 & Se acepta \\
\hline $\mathrm{H} 8$ & Precio & Lealtad comportamental & 0.43 & Se acepta \\
\hline $\mathrm{H} 9$ & Precio & Lealtad cognitiva & 0.33 & Se acepta \\
\hline $\mathrm{H} 10$ & Precio & Lealtad afectiva & 0.31 & Se acepta \\
\hline $\mathrm{H} 11$ & Calidad & Lealtad comportamental & 0.46 & Se acepta \\
\hline
\end{tabular}




\begin{tabular}{lllll}
\hline Hipótesis & & Relación positiva entre & Correlación & Conclusión \\
\hline $\mathrm{H} 12$ & Calidad & Lealtad afectiva & 0.39 & Se acepta \\
\hline $\mathrm{H} 13$ & Valor utilitario & Lealtad comportamental & 0.35 & Se acepta \\
\hline $\mathrm{H} 14$ & Valor utilitario & Lealtad cognitiva & 0.26 & Se acepta \\
\hline $\mathrm{H} 15$ & Valor utilitario & Lealtad afectiva & 0.31 & Se acepta \\
\hline
\end{tabular}

Fuente: elaboración propia.

Finalmente, la figura 1 da razón de lo presentado en la tabla 3.

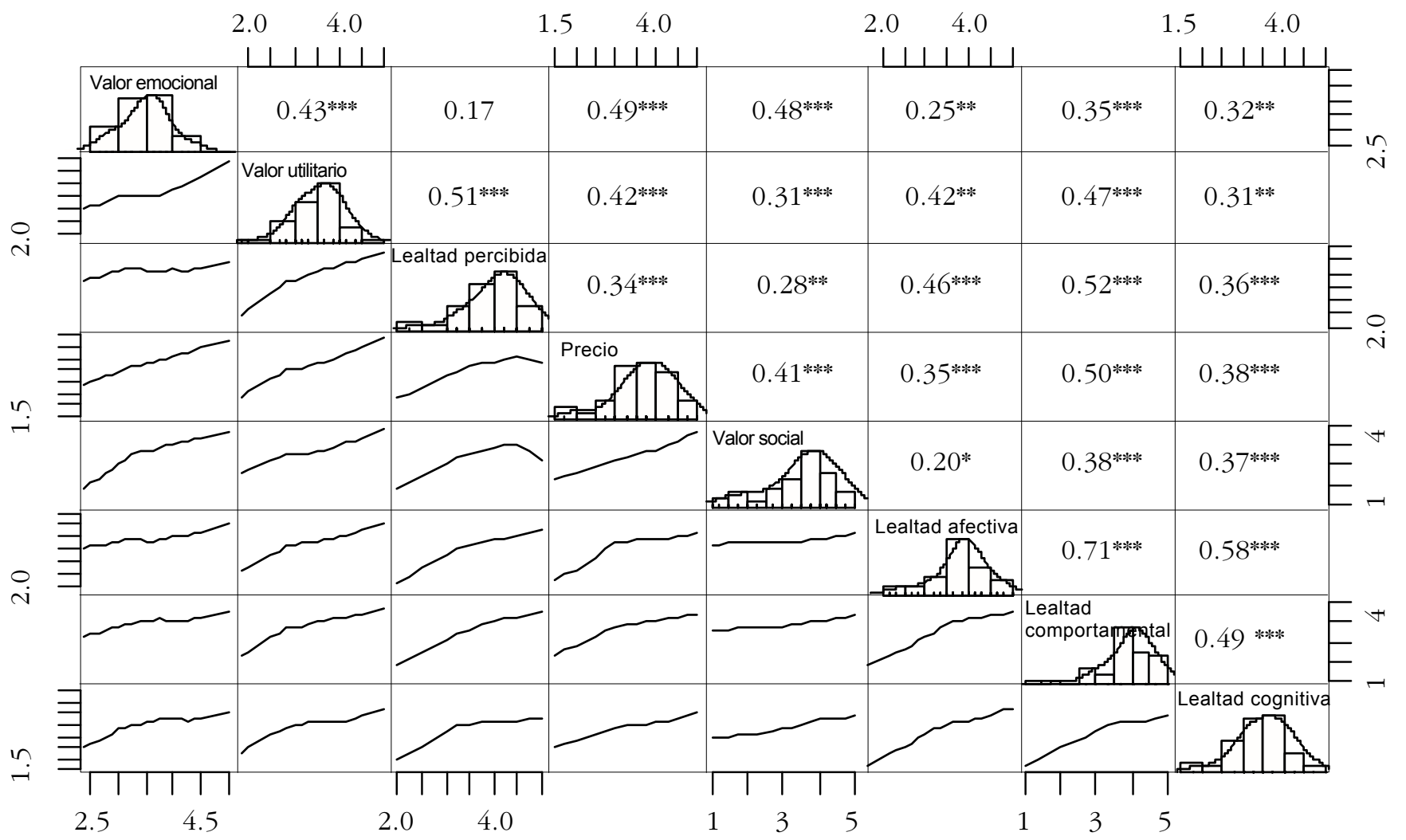

Figura 1. Correlaciones entre las dimensiones de valor percibido y lealtad de marca

Nota: la figura muestra la distribución de cada variable en diagonal. En la parte inferior de la diagonal se muestran los gráficos de dispersión bivariados con una línea ajustada. En la parte superior de la diagonal se evidencia el valor de la correlación más el nivel de significación como estrellas. Teniendo en cuenta que cada nivel de significación está asociado a un símbolo, valores de $p(0)$ corresponden al símbolo ***, valores de $p$ (0.001) corresponden al símbolo ** y valores de $p$ (1) no tienen asignados simbología.

Fuente: elaboración propia. 


\section{Discusión}

Los resultados determinan una relación entre los factores de valor percibido y los de lealtad, lo que constituía el objetivo principal de la investigación. Se validaron todas las hipótesis planteadas. Así, para el caso de tarjetas de crédito de marca compartida, es evidente que para los clientes estas alianzas conllevan respuestas más positivas, lo que va en línea con lo planteado por Swaminathan et al. (2015), ya que en general hay una alta frecuencia de uso para comprar en la marca de ropa de la alianza, respectivamente.

De igual manera, la presente investigación está en consonancia con lo planteado por Kim et al. (2007), en cuanto a que el cobranding pretende obtener lealtad en los clientes para el producto resultado de la alianza, así como para las marcas de forma individual; para este caso, se evidencia existencia de un nivel de lealtad medioalto hacia la marca de ropa, accesorios y calzado. Respecto al valor percibido, también se está en línea con lo planteado por Helmig et al. (2008): se evidencia una generación de valor percibido (nivel medioalto) en los clientes en relación con la tarjeta de crédito de marca compartida.

La calidad percibida obtuvo el nivel más alto dentro de los factores de valor percibido, lo que indica, según Petrick (2002) y Walsh et al. (2014), que el producto que se adquiere o se usa, en este caso, la tarjeta de crédito de marca compartida está bien constituida, es confiable y brinda lo esperado, lo cual es claro en los resultados de los ítems que midieron la calidad percibida, ya que se obtuvieron medias de 4.08 para "La tarjeta de crédito de marca compartida le brinda el servicio que necesita", de 4.14 para "La tarjeta de crédito de marca compartida cumple sus necesidades", de 4.15 para "Se siente seguro al hacer transacciones con la tarjeta de crédito de marca compartida" y de 3.98 para "La tarjeta de crédito de marca compartida brinda mejores prestaciones que otras tarjetas de crédito".

La lealtad comportamental obtuvo el nivel más alto dentro de los factores de lealtad, lo que indica, según Jacoby y Kiner (1973, citados en Ramírez Angulo \& Duque Oliva, 2013), que existe alta frecuencia de compra y recompra; en este caso en la marca de ropa, lo cual es claro en los resultados de los ítems que midieron la lealtad comportamental, ya que se obtuvieron medias de 3.93 para "Fue muy importante comprar esta marca más que cualquier otra", de 3.92 para "Me siento consistente comprando esta marca más que cualquier otra" y de 3.81 para 
"Una vez que he elegido una marca en particular me mantengo en mi elección”. Por otra parte, los resultados están en la misma línea de lo mencionado por Mencarelli y Lombart (2017), en cuanto a que el valor percibido es un predictor de lealtad comportamental, ya que para este caso se validan las hipótesis 1, 4, 7, 10 y 13.

Particularmente, para este estudio no se presenta lo mencionado por Rundle-Thiele (2005) y Söderlund (2006), citados por García y Fernández (2016), respecto a que es posible que cada consumidor presente estados de lealtad en mayor o menor grado para cada una de las dimensiones, es decir, que por ejemplo se evidencie en un consumidor alta fidelidad comportamental pero baja actitudinal, ya que para los factores de lealtad estudiados todos tienen niveles consecuentes entre sí, considerándose como medioaltos.

\section{Conclusiones}

Este estudio se considera que aporta a reducir el vacío que se encuentra en cuanto a la falta de evidencia de investigación empírica sobre la efectividad de la marca compartida, como lo afirman Wang y Hsu (2016). Así, en vista de que no hay antecedentes empíricos referentes a tarjetas de crédito de marca compartida, esta investigación se constituye como referente y pionera al respecto, al menos en lo respectivo a las alianzas entre establecimientos bancarios y marcas de ropa, accesorios y calzado, y complementa estudios referentes a aerolíneas, tal como se mencionaron anticipadamente.

La principal limitación de la investigación se encuentra en el tipo de muestreo seleccionado, porque es uno no probabilístico, seleccionado por el hecho no contar con acceso a la base de datos oficial sobre clientes de tarjetas de crédito de marca compartida vigentes en Bogotá (Colombia).

A partir de este trabajo, se sugieren futuras líneas de investigación que se orienten a la medición de otros constructos para el caso de las tarjetas de crédito de marca compartida, por ejemplo, las actitudes, el involucramiento, entre otras, tanto en términos del producto cobrand en sí mismo como de los impactos en las marcas vinculadas. De igual manera, 
son importantes estudios en torno a otros tipos de tarjetas de crédito de marca compartida, por ejemplo, con aerolíneas, restaurantes, servicios de telefonía móvil, cooperativas, entre otras, con el fin de realizar contrastes y derivar conclusiones al respecto.

\section{Referencias}

Babin, B., Darden, W. R., \& Griffin, M. (1994). Work and/or fun: Measuring hedonic and utilitarian shopping value. Journal of Consumer Research, 20(4), 644-656.

Bolton, R. N., \& Lemon, K. N. (1999). A dynamic model of customers' usage of services: Usage as an antecedent and consequence of satisfaction. Journal of Marketing Research, 36(2), 171-186.

Bravo, R., \& Pina, J. (2009). Las alianzas de marca online-offline: Una estrategia de entrada al mercadeo offline. Cuadernos de Ciencias Económicas y Empresariales, (56-57), 39-60. http://cuadernos.uma.es/pdfs/pdf689.pdf

Ching Wang, S., Soesilo, P., \& Zhang, D. (2015). Impact of luxury brand retailer cobranding strategy on potential customers: A cross-cultural study. Journal of International Consumer Marketing, 27(3), 237-252.

Colmenares, O., \& Saavedra, J. (2007). Aproximación teórica de la lealtad de marca: Enfoques y valoraciones. Cuadernos de Gestión, 7(2), 69-81.

Dahlstrom, R., \& Conway Dato-On, M. (2004). Business-to-business antecedents to retail cobranding. Journal of Business-to-Business Marketing, 11(3), 1-22.

Dedeoglu, B. B., Bilgihan, A., Ye, B. H., Buonincontri, P., \& Okumus, F. (2018). The impact of servicescape on hedonic value and behavioral intentions: The importance of previous experience. International Journal of Hospitality Management, 72, 10-20. https://doi. org/10.1016/j.ijhm.2017.12.007

ElBoghdady, D. (2003, 14 de octubre). Banks, retailers again push 'co-branded' credit cards. The Washington Post. https://www.washingtonpost.com/archive/business/2003/10/14/banksretailers-again-push-co-branded-credit-cards/6a52e7bf-2a8d-4322-a172-61390b554db2/

El Universal (2016, 21 de julio). El uso de las tarjetas de crédito y débito sigue creciendo en Latinoamérica. https://www.eluniversal.com.co/economica/el-uso-de-las-tarjetas-de-credito-y-debito-sigue-creciendo-en-latinoamerica-230990-JQeu337583

García, C., \& Fernández, M. (2016). Desarrollo y validación inicial de una escala para evaluar la fidelidad de los consumidores hacia las tiendas. European Research on Management and Business Economics, 22(2), 94-100. https://doi.org/10.1016/j.iedee.2015.10.005 
Grace, D.A., \& O'Cass, A. (2005). Examining the effects of service brand communications on brand evaluation. Journal of Product and Brand Management, 14(2), 106-116.

Grębosz, M. (2012). The outcomes of the cobranding strategy. Chinese Business Review, 11(9), 823-829. http://www.davidpublisher.com/Public/uploads/Contribute/5514c3d22e092.pdf

Grębosz-Krawczyk., \& Pointet, J.-M. (2017). Co-branding strategy as a source of innovation on international market. Journal of Intercultural Management, 9(3), 63-77. https://doi. org/10.1515/joim-2017-0014

Helmig, B., Huber, J., \& Leeflang, P. (2008). Co-branding: The state or the art. Schmalenbach Business Review, 60, 359-377.

Holbrook, M., \& Hirschman, E. (1982). Hedonic consumption: Emerging concepts, methods and propositions. Journal of Marketing, 46(3), 92-101.

Hoyos, R. (2016). Branding: El arte de marcar corazones. Ecoe.

Jiang, K., Luk, S. T., \& Cardinali, S. (2018). The role of pre-consumption experience in perceived value of retailer brands: Consumers' experience from emerging markets. Journal of Business Research, 86, 374-385. https://doi.org/10.1016/j.jbusres.2017.09.026

Kapferer, J. (2008). The new strategic brand management: Creating and sustaining brand equity long term (4. ${ }^{a}$ ed.). Kogan Page.

Kim, W., Lee, S., \& Lee, H. (2007). Cobranding and brand loyalty.Journal of Quality Assurance in Hospitality and Tourism, 8(2), 1-23.

Kim, M. J., Lee, C. K., Petrick, J. F., \& Hahn, S. S. (2018). Factors affecting international event visitors' behavioral intentions: the moderating role of attachment avoidance. Journal of Travel and Tourism Marketing, 35(8), 1027-1042.

Mencarelli, R., \& Lombart, C. (2017). Influences of the perceived value on actual repurchasing behavior: Empirical exploration in a retailing context.Journal of Retailing and Consumer Services, 38, 12-21. https://doi.org/10.1016/j.jretconser.2017.04.008

Nguyen, N. (2018). The combined effects of service offering and service employees on the perceived corporate reputation. Athens Journal of Business and Economics, 4(2), 129-146.

Oeppen, J., \& Jamal, A. (2014). Collaborating for success: Managerial perspectives on cobranding strategies in the fashion industry. Journal of Marketing Management, 30(9-10), 925-948.

Park, W., Jun, S., \& Shocker, A. (1996). Composite branding alliances: An investigation of extension and feedback effects. Journal of Marketing Research, XXXIII, 453-466.

Petrick, J. F. (2002). Development of a multi-dimensional scale for measuring the perceived value of a service. Journal of Leisure Research, 34(2), 119-134. 
Ramírez Angulo, P. J., \& Duque Oliva, E. J. (2013). Involucramiento de producto y lealtad de marca para productos de consumo masivo en Bogotá D.C. Estudios Gerenciales, 29(128), 303-312. https://doi.org/10.1016/j.estger.2013.09.004

Rúmbolo, A. (2015). Co-branding y moda: Una estrategia de co-creación de valor [tesis de pregrado]. Universidad de San Andrés, Buenos Aires.

Shen, B., Jung, J., Chow, P., \& Wong, S. (2014). Co-branding in fast fashion: The impact of consumers' need for uniqueness on purchase perception. En T. M. Choi (Ed.), Fashion branding and consumer behaviors (pp. 101-112). Springer.

Shen, B., Choi, T. S., \& Chow, P.S. (2017). Brand loyalties in designer luxury and fast fashion co-branding alliances. Journal of Business Research, 81, 173-180. https://doi.org/10.1016/j. jbusres.2017.06.017

Sheth, J. N., Newman, B. I., \& Gross, B. L. (1991). Why we buy what we buy: A theory of consumption values. Journal of Business Research, 22(2), 159-170.

Simonin, B., \& Ruth, J. (1998). is a company known by the company it keeps? Assessing the spillover effects of brand alliances on consumer brand attitudes. Journal of Marketing Research, $\mathrm{x} x \mathrm{x}$, , 30-42.

Superintendencia Financiera de Colombia. (2019). Informe de tarjetas de crédito y débito. https://www.superfinanciera.gov.co/inicio/informe-de-tarjetas-de-credito-y-debito-60952

Swaminathan, V., Gürhan, Z., Kubat, U., \& Hayran, C. (2015). How, when, and why do attribute-complementary versus attribute-similar cobrands affect brand evaluations: A concept combination perspective. Journal of Consumer Research, 42(1), 45-58.

Sweeney, J. C., \& Soutar, G. N. (2001). Consumer perceived value: The development of a multiple item scale. Journal of Retailing, 77(2), 203-220.

Tur Viñes, V. (2012). Co-branding, creatividad y crisis. Creatividad y Sociedad, (xviII), 1-17.

Vera, J., \& Trujillo, A. (2009). El papel de la calidad del servicio del restaurante como antecedente de la lealtad del cliente. Panorama Socioeconómico, 27(38), 16-30.

Walsh, G., Shiu, E., \& Hassan, L. M. (2014). Replicating, validating, and reducing the length of the consumer perceived value scale. Journal of Business Research, 67(3), 260-267.

Wang, S., \& Hsu, M. (2016). Airline co-branded credit cards-An application of the theory of planned behavior. Journal of Air Transport Management, 55, 245-254.

Wang, S., \& Farquhar, J. (2018). Co-branded services: perceived benefits and involvement of co-branded credit cards. International Journal of Bank Marketing, 36(5), 969-987.

Wright, O., Frazer, L., \& Merrilees, B. (2007). McCafe: The McDonald's cobranding experience. Journal of Brand Management, 14(6), 442-457. 
Yang, J., Gu, Y., \& Cen, J. (2011). Festival tourists' emotion, perceived value, and behavioral intentions: A test of the moderating effect of festivalscape. Journal of Convention and Event Tourism, 12(1), 25-44.

Zeithaml, V. A. (1988). Consumer perceptions of price, quality, and value: A means-end model and synthesis of evidence. Journal of Marketing, 52(3), 2-22. 